\title{
Evaluation and Optimization of College English Teaching Effect Based on Improved Support Vector Machine Algorithm
}

\author{
Zhang Bao-feng $(\mathbb{C}$ \\ Shangqiu Institute of Technology, Shangqiu, Henan 476000, China \\ Correspondence should be addressed to Zhang Bao-feng; 1350013010@sqgxy.edu.cn
}

Received 14 December 2021; Revised 31 December 2021; Accepted 5 January 2022; Published 28 January 2022

Academic Editor: Tongguang $\mathrm{Ni}$

Copyright (c) 2022 Zhang Bao-feng. This is an open access article distributed under the Creative Commons Attribution License, which permits unrestricted use, distribution, and reproduction in any medium, provided the original work is properly cited.

Improved SVM algorithm improves the efficiency of College English teaching effect evaluation and meets the requirements of College English teaching evaluation. Based on the relevant theories, this paper constructs the evaluation index system with teachers and students as the main body and takes the questionnaire survey results as the input samples of the LSSVM algorithm. Compared with the evaluation accuracy of an optimized BP neural network and the category weighted gray target decisionmaking method, the results show that the evaluation accuracy of optimized LSSVM algorithm is $96.26 \%$. Taking SIT as an example, this paper uses the optimized LSSVM algorithm to evaluate its teaching effect and obtains that teachers' literature and teaching contents are important factors to improve the effect of English teaching. Therefore, this paper introduces the intelligent voice system to optimize the English teaching design of SIT. The teaching design is optimized from the dimensions of teaching objectives, learning situation, teaching content, teaching media and curriculum materials, and teaching procedures.

\section{Introduction}

Teaching effect evaluation has become a hot issue in the field of education at home and abroad. Teaching effect is the core of school survival and development. Teaching evaluation is a very important and indispensable part of the teaching process and an important means to test the teaching effect. Traditional teaching evaluation methods only focus on students' learning effect, which is not conducive to the reform of English teaching methods and contents.

English teaching effect evaluation refers to the evaluation of teachers' teaching effect from the aspects of teaching content, subject organization, teaching achievement, and learning interest. Through the evaluation, the direction of curriculum optimization can be put forward. Eleni et al. (2012) believe that teaching motivation and teaching satisfaction are the main factors of curriculum teaching effect and put forward effective evaluation methods for teaching motivation, such as the experimental method, questionnaire method, and text analysis method [1]. The traditional questionnaire survey and observation evaluation cannot objectively evaluate the classroom quality for a long time and cannot obtain clear evaluation results. In this case, Li et al. (2019) propose an intelligent school teacher quality evaluation platform. By considering the sensor information, flow information, and power information of mobile devices in the teaching process, the teacher quality evaluation score is given through the neural network training classification model [2]. Nalla (2019) proposes an evidence-based teaching strategy evaluation framework [3]. Leong et al. (2012) discuss the method of analyzing text mining of short message service (SMS) in teaching evaluation, and analyze and classify the information [4]. Ahmad (2018) analyzes how to the improve students' response rate to teaching curriculum evaluation through the survey results. The survey results show that the effective communication of teachers can effectively improve students' participation in the evaluation of teaching curriculum quality [5]. Cadez et al. (2017) analyze the relationship between learning style and academic achievement of accounting students through teacher evaluation [6]. Bi (2018) studies the teaching evaluation grade and uses the evaluation based on statistical process control theory and standard deviation diagram to evaluate the school teaching grade [7]. 
In the selection of evaluation methods, the emotion classification model is widely used. Mullen and Collier (2004) first analyze the syntax between texts and then further analyze the emotion of the text based on the SVM algorithm [8]. Moraes et al. (2013) integrate the machine learning algorithm and deep learning technology into teaching effect evaluation, compare the classification effect of the support vector machine algorithm with that of the artificial neural network algorithm, and prove that the classification effect of ANN is equivalent to that of SVM [9]. Besides, Fuzzy mathematics theory, analytic hierarchy process, and BP neural network are also commonly used in the evaluation of CETE. Kennedy and Eberhart (1997) proposed an evolutionary algorithm to solve discrete problems based on the study of bird foraging behavior. Compared with the genetic algorithm, it has faster convergence speed in solving multiuser detection problems [10].

Sun (2020) et al. have developed an online English teaching assistant system, which uses the decision tree algorithm and a neural network to generate an English teaching evaluation model based on decision tree technology and studies the potential relationship between the evaluation results and various factors [11]. However, optimizing the evaluation model of college teaching quality based on the BP neural network will ignore the factors of teaching quality, resulting in the low accuracy of CETE evaluation. Krohling and de Souza (2012) define the positive and negative target distance from each scheme to the positive and negative ideal scheme, and sort the schemes according to the comprehensive target distance of each scheme [12]. The teaching evaluation method based on category weighted gray target decision making has low evaluation efficiency due to the complex calculation process. In the application of the intelligent system to curriculum teaching, the student model of the intelligent teaching system based on the Bayesian network established by Lan (2020) can not only objectively evaluate students' cognitive ability but also infer students' next learning behavior [13]. Myers (2021) uses the intelligent teaching system to automatically detect students' emotional state and guide students into a positive learning state [14]. Ramadan and Vasilakos (2017) collected shallow brain activity signals, extracted specific brain waves according to specific frequencies, and evaluated students' attention, emotion, and cognitive load [15].

The existing CETE research mainly focuses on the selection of the evaluation model and the improvement of model accuracy, and there is less research on practical application and optimization means. This paper not only optimizes the evaluation model but also innovatively introduces information technology means such as the intelligent voice system to improve the level of College English teaching. Based on the past research, this paper finds that mathematical algorithms are widely used in the evaluation of CETE, but most of them only use one method, and the optimization of the algorithm needs to be further improved. In this paper, the SVM algorithm is used as the evaluation method of CETE, and the PSO algorithm is used to optimize the SVM algorithm.
This paper constructs the evaluation index system from the perspectives of teachers and students and fully considers students' interests and teachers' feelings. By comparative analysis, the algorithm with the highest accuracy is selected as the evaluation method of this paper, and the selected evaluation method is applied to practice to obtain the most important influencing factors. The intelligent voice system is introduced to optimize College English teaching curriculum design, the improvement of teaching evaluation quality is fully considered, and curriculum design optimization is carried out while improving students' English learning achievements and considering students' interests and learning needs. In the evaluation process of this paper, the effect of College English teaching is divided into four levels: excellent, good, pass, and fail, and the optimized algorithm is used for empirical research. Firstly, this paper selects the Shanghai Institute of Technology for a case study to evaluate its College English teaching effect. Secondly, according to the evaluation results of each index and the overall evaluation results, the most important index in the process of curriculum optimization design is obtained. Finally, the intelligent speech system is introduced to formulate the optimization scheme and suggestions of College English teaching curriculum design.

\section{Evaluation Model of College English Teaching Effect}

2.1. Evaluation Index System of College English Teaching Effect. There are many theories for the evaluation system of College English teaching effect. Teachers and students are the two main bodies of the classroom, and they participate in the whole process of teaching. Therefore, starting from these two subjects, CETE can be evaluated more comprehensively. According to the relevant theories, an evaluation index system with teachers and students as the theme is constructed, as shown in Figure 1. The evaluation index system constructed in this paper can evaluate the teaching effect more comprehensively from the main perspective of students and teachers and from the different perspectives of participants in teaching activities. From the perspective of students, the evaluation index mainly comes from their feelings in class. Therefore, the evaluation indicators from the perspective of students mainly include course content, class atmosphere, teaching order, teaching skills, sense of responsibility, and interest. From the perspective of teachers, the evaluation indicators mainly come from the actual teaching effect and the number of courses. Therefore, the evaluation indicators from the perspective of teachers mainly include students' performance, correcting homework, the number of classes dismissed in advance, the number of classes transferred, and the number of classes suspended. The interpretation and definition of indicators are shown in Table 1.

Teachers' sense of responsibility and English achievement are important indicators to evaluate the effect of College English teaching. The evaluation index data of College English teaching effect are used as the input sample of the least squares support vector machine to realize the evaluation of College English teaching effect. 


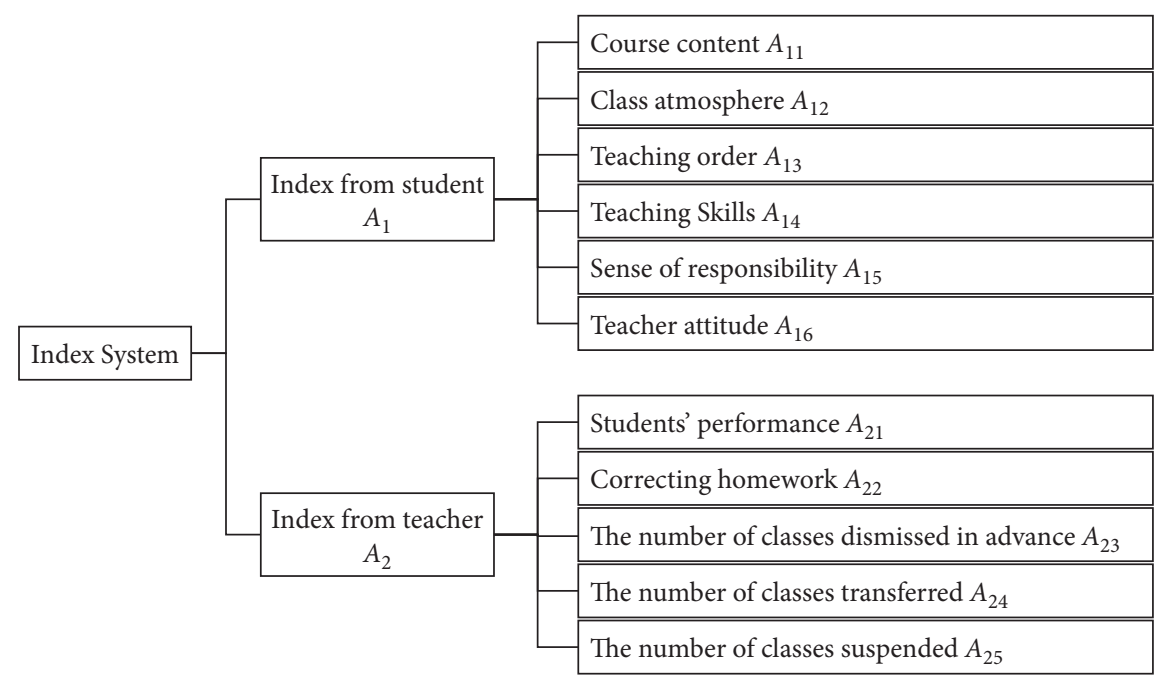

Figure 1: Evaluation index system of CETE.

TABLE 1: English teaching effect evaluation index.

\begin{tabular}{lcc}
\hline & Index & Explanation \\
\hline$A_{11}$ & Course content & Reasonableness of English subject content \\
$A_{12}$ & Class atmosphere & The quality of English topic atmosphere \\
$A_{13}$ & Teaching order & The organization and hierarchy of lectures \\
$A_{14}$ & Teaching skills & Teachers' teaching skills \\
$A_{15}$ & Sense of responsibility & Teachers' sense of responsibility to students \\
$A_{16}$ & Teacher attitude & Thes of teachers in correcting homework \\
$A_{21}$ & Students' performance & Therionts performance in class \\
$A_{22}$ & Correcting homework & Accuracy of operation \\
$A_{23}$ & The number of classes dismissed in advance & The teacher dismissed the class early for reasons other than the course \\
$A_{24}$ & The number of classes transferred & Teachers change classes for reasons other than the course \\
$A_{25}$ & The number of classes suspended & Teachers do not attend classes for reasons other than the course \\
\hline
\end{tabular}

2.2. Evaluation Principle of LSSVM. This paper uses the least squares support vector machine (LSSVM) method to evaluate English language teaching. LSSVM is one of the most widely used methods with good accuracy and precision in nonlinear signal processing. Compared with artificial neural networks, it can overcome the disadvantages of long training time, randomness of training results, and overlearning [16]. Compared with SVM, LSSVM transforms inequality constraints into equation constraints and quadratic programming problems into a linear system of equation problems, which greatly facilitates the solution of Lagrange multipliers $\lambda$. The computational efficiency of LSSVM is higher than that of SVM. Furthermore, LSSVM is equally suitable for classification and regression tasks in high-dimensional input spaces and can be extended to unsupervised learning and recurrent neural networks.

The main description of the CETE evaluation model used in this paper is as follows: a nonlinear mapping $\varphi\left(\mathbf{x}_{\mathbf{i}}\right)$ is chosen to map the sample vector with $\mathrm{n}$-dimensional input data as $x$ and one-dimensional output data as $y$ $\left[\left(x_{1}, y_{1}\right), \ldots,\left(x_{i}, y_{i}\right), \quad \mathbf{x}_{\mathbf{i}} \in \mathbf{R}^{n}, y_{i} \in \mathbf{R}, \quad i=1,2, \ldots\right.$, $m$ ], from the original space to the high-dimensional feature space. The regression equation of LSSVM is shown in the following equation:

$$
y_{i}=f\left(\mathbf{x}_{\mathbf{i}}\right)=\boldsymbol{\omega}^{T} \cdot \varphi\left(\mathbf{x}_{\mathbf{i}}\right)+b .
$$

In the above equation, $\omega$ is the weight vector and the constant $b$ is the bias term. The optimization problem of LSSVM is shown in the following equation:

$$
\min \frac{1}{2} \boldsymbol{\omega}^{T} \cdot \boldsymbol{\omega}+\gamma \cdot \frac{1}{2} \sum_{i=1}^{m} \xi_{i}^{2} .
$$

In the above equation, $\gamma$ is the regularization parameter and $\xi$ is the error variable. Compared to SVM, the constraints of LSSVM are different, as shown in the following equation:

$$
y_{i}=\boldsymbol{\omega}^{T} \cdot \varphi\left(\mathbf{x}_{\mathbf{i}}\right)+b+\xi_{i}^{2}, i=1,2, \ldots, m .
$$

The inequality constraint of the SVM is replaced by an equation constraint, and the Lagrange function is constructed for the solution, as shown in the following equation:

$$
\begin{aligned}
L(\boldsymbol{\omega}, b, \xi, \lambda)= & \frac{1}{2} \boldsymbol{\omega}^{T} \cdot \boldsymbol{\omega}+\gamma \cdot \frac{1}{2} \sum_{i=1}^{m} \xi_{i}^{2} \\
& -\sum_{i=1}^{m} \lambda_{i}\left[\boldsymbol{\omega}^{T} \cdot \varphi\left(\mathbf{x}_{\mathbf{i}}\right)+b+\xi_{i}-y_{i}\right] .
\end{aligned}
$$


In the above equation, $\lambda_{i}$ is the Lagrange multiplier. The optimal value is calculated under the Karush-Kuhn-Tucker (KKT) optimization condition. The equation constraint is shown in the following equation:

$$
\left\{\begin{array}{l}
\frac{\partial L}{\partial \boldsymbol{\omega}}=0 \longrightarrow \omega=\sum_{i=1}^{m} \lambda_{i} \varphi\left(\mathbf{x}_{\mathbf{i}}\right) \\
\frac{\partial L}{\partial b}=0 \longrightarrow \sum_{i=1}^{m} \lambda_{i}=0 \\
\frac{\partial L}{\partial \xi}=0 \longrightarrow \lambda_{i}=\gamma \xi_{i} \\
\frac{\partial L}{\partial \lambda}=0 \longrightarrow \boldsymbol{\omega}^{T} \cdot \varphi\left(\mathbf{x}_{\mathbf{i}}\right)+b+\xi_{i}-y_{i}=0
\end{array}\right.
$$

By eliminating $\boldsymbol{\omega}$ and $\xi$ in the above equation, the quadratic optimization problem is replaced by solving a linear system of equations, and the result is calculated as in the following equation:

$$
\left[\begin{array}{c}
0 \\
\mathbf{Y}
\end{array}\right]=\left[\begin{array}{cc}
0 & \mathbf{Q}^{T} \\
Q & \mathbf{\Omega}+\gamma^{-1} \mathbf{I}
\end{array}\right]\left[\begin{array}{l}
b \\
\lambda
\end{array}\right] .
$$

In the above equation, $\mathbf{I}$ is the identity matrix; $\alpha$ is a support vector; $\mathbf{Y}=\left[y_{1}, y_{2}, \ldots, y_{m}\right]^{T} ; \mathbf{Q}=[1,1, \ldots, 1]^{T} ; \boldsymbol{\Omega}$ represents the inner product of the kernel matrix, $\boldsymbol{\Omega} \in \mathbf{R}^{m \times m}$, and $\boldsymbol{\Omega}_{i j}=\varphi\left(\mathbf{x}_{\mathbf{i}}\right)^{T} \varphi\left(\mathbf{x}_{\mathbf{j}}\right)=K\left(\mathbf{x}_{\mathbf{i}}, \mathbf{x}_{\mathbf{j}}\right), \quad i, j=1,2, \ldots, m$. Based on the kernel function in the original space, the regression of LSSVM is obtained function for the following equation:

$$
y=\sum_{i=1}^{m} \lambda_{i} K\left(\mathbf{x}_{\mathbf{i}}, \mathbf{x}_{\mathbf{j}}\right)+b .
$$

To circumvent the dimensional catastrophe, a radial basis kernel function commonly used in LSSVM is introduced instead of the inner product operation in the highdimensional feature space. The radial basis kernel function is shown in the following equation:

$$
K\left(\mathbf{x}_{\mathbf{i}}, \mathbf{x}_{\mathbf{j}}\right)=\exp \left(-\frac{\left(x-x_{i}\right)^{2}}{\sigma^{2}}\right) .
$$

In the above equation, $\sigma$ represents the kernel function width. The generalization ability of LSSVM can be enhanced by optimizing two parameters, $\gamma$ and $\sigma$. When LSSVM is used for University English teaching evaluation, the particle swarm algorithm can be used for the parameter finding problem, and the specific evaluation process is shown in Figure 2.

\subsection{Parameter Optimization Based on PSO. Parameter se-} lection is an important research direction in the field of LSSVM research. Many methods can be used to determine $\gamma$ and $\sigma$, but there are deficiencies in memory and time. Particle swarm optimization (PSO) algorithms have the advantages of being less prone to local minima, easy to implement, and with fewer tuning parameters. Thus, PSO is often used to solve nonlinear, nonintegrable, and multimodal problems. In PSO, each potential solution to the optimization problem is called a "particle." Each particle has its own stance and velocity (which determines its direction of flight and distance) and an adaptation value determined by the function being optimized.

A population of $m$ particles is set up in a $d$-dimensional search space, where the optimal position of particle $i$ searched in the space is described by $\mathbf{p}_{\mathbf{i}}$, $\mathbf{p}_{\mathbf{i}}=\left[p_{i 1}, p_{i 2}, \ldots, p_{i d}\right]$; the velocity is described by $\mathbf{v}_{\mathbf{i}}$, $\mathbf{v}_{\mathbf{i}}=\left[v_{i 1}, v_{i 2}, \ldots, v_{i d}\right]$; the position of particle $i$ is described by $\mathbf{x}_{\mathbf{i}}, \mathbf{x}_{\mathbf{i}}=\left[x_{i 1}, x_{i 2}, \ldots, x_{i d}\right]$; the optimal position searched for in the total population is described by $\mathbf{p}_{\mathbf{g}}$, $\mathbf{p}_{\mathbf{g}}=p_{g 1}, p_{g 2}, \ldots, p_{g d}$. The method to update the particle position and velocity is given in the following equations:

$$
\begin{aligned}
& x_{i d}^{k+1}=x_{i d}^{k}+v_{i d}^{k+1}, \\
& v_{i d}^{k+1}=\omega v_{i d}^{k}+c_{1} r_{1}\left(p_{i d}-x_{i d}^{k}\right)+c_{2} r_{2}\left(p_{g d}-x_{i d}^{k}\right) .
\end{aligned}
$$

Among them, $\omega$ is the inertia weight coefficient; $c_{1}$ and $c_{2}$ are the acceleration constants; $k$ is the optimization algebra; $v_{i d}^{k}$ and $x_{i d}^{k}$ are the search velocity and position of the particle in the space at algebra $k ; r_{1}$ and $r_{2}$ are the random numbers. The process of optimizing the parameters $\gamma$ and $\sigma$ by PSO is as follows:

(1) Normalize and preprocess the CETE evaluation data

(2) Set the parameter values

(3) Initialize processing

(4) Set the individual extreme value $P_{i \text { best }}$ and the global extreme value $g_{\text {best }}$, and calculate the adaptation value of each particle according to its current position, $f=\sum_{i=1}^{N}\left|y_{i}-y_{1}^{\prime}\right|$, where $N$ is the total number of samples

(5) In order to generate new populations, update the particle positions and velocities according to equations (9) and (10)

(6) Solve for the fitness value $f$ for each particle within the new population

(7) Compared with the previous optimal speed and optimal position of the population, if it is excellent, the conversion will be implemented, otherwise, no adjustment will be made

(8) If the optimal ending condition is not met, we need to make the number of iterations $t=t+1$ and return to Step 4 to find the optimal solution

2.4. Evaluation Process. Combined with the evaluation index system of College English teaching effect, a College English teaching effect evaluation model based on the particle swarm optimization algorithm and support vector machine is established. The flowchart of the model is shown in Figure 3.

The input sample of LSSVM is the value of CETE evaluation data, which is from the expert rating. And the evaluation results are obtained through the operation of the 


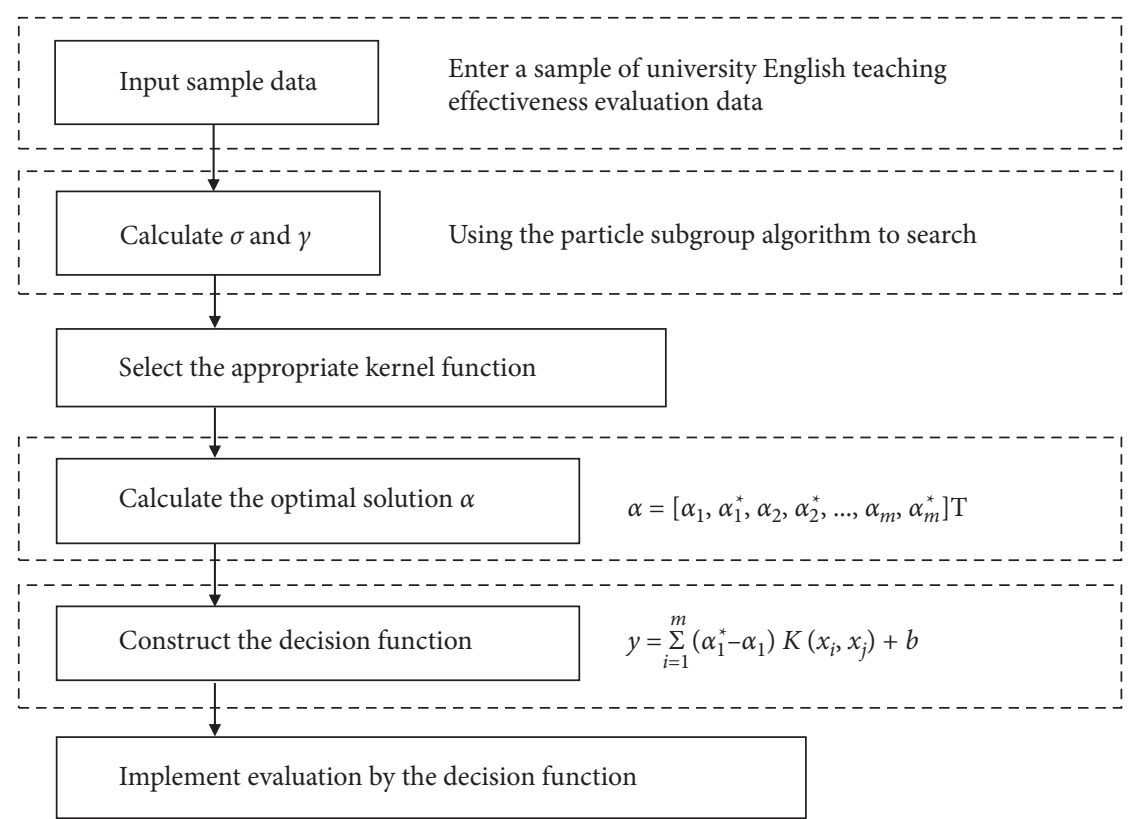

FIGURE 2: Evaluation model main process.

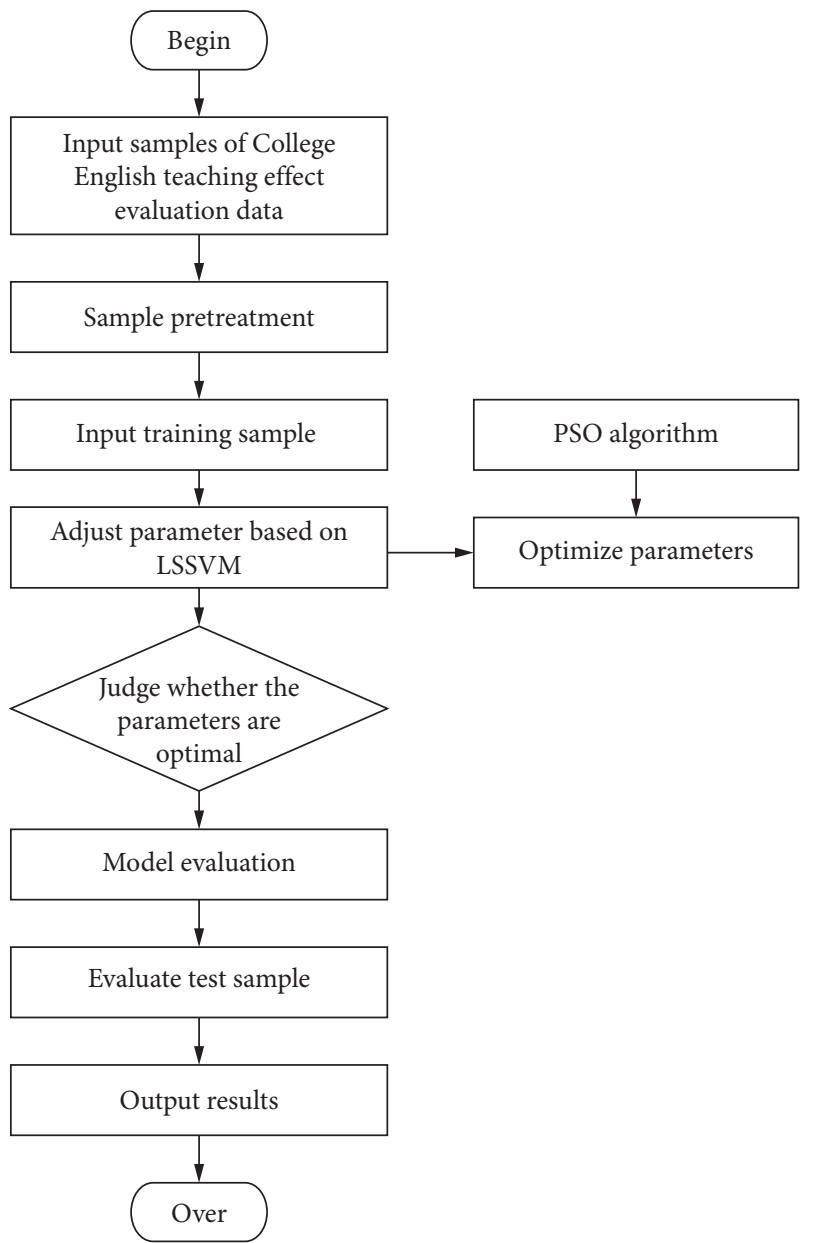

Figure 3: Flowchart of CETE based on the improved support vector machine algorithm.
SVM algorithm. This paper optimizes the evaluation algorithm by comparing the evaluation results before and after adding the PSO algorithm. The PSO algorithm mainly solves the optimal solutions of kernel function parameters and regularization parameters of LSSVM, and then optimizes the process of CETE evaluation. It judges whether the existing parameters are the optimal solution through the IF function. If the result is yes, the test samples are evaluated, and the results are output. If the result is no, the particle swarm optimization algorithm needs to be used to readjust the parameters to the optimal solution. The above process is a machine learning process, and the computer realizes the purpose of automatic tuning through its own learning.

\section{Experimental Results and Analysis}

This paper selects the English classroom teaching effect of Shangqiu Institute of Technology as the research object and collects sample data according to the evaluation index of CETE. Through the actual situation of College English teaching and the evaluation of College English teaching effect by experts, we can get the quality level of College English classroom teaching and 200 data samples for testing. In the model building process, this paper chooses the Gaussian radial basis function as the kernel function.

The training data of each degree of the six faults are input to the LSSVM regressor for learning, while the PSO algorithm is used for multiobjective optimization of the regularization parameters and kernel function parameters. The population size of PSO is set to 200, the learning factor to 1.49 , the maximum number of iterations to 1000 , and the inertia weight to 1 initially. The final choice of the best model parameters is $\left(\gamma, \sigma^{2}\right)=(2316.57,224.46), b=9.93$. The 
regression performance of the model is evaluated by the ratio of the number of correct model predictions to the total number of samples, as shown in the following equation:

$$
\text { accuracy }=\frac{\mathrm{TP}+\mathrm{TN}}{\mathrm{TP}+\mathrm{FP}+\mathrm{FN}+\mathrm{TN}} .
$$

In order to verify the effectiveness of the PSO-LSSVM method for CETE assessment, we also use the BP neural network [17] and gray target decision method [18] for comparison testing. Three methods are used to test the experimental dataset to obtain their accuracy calculation results, as shown in Table 2 and Figure 4. As can be seen from Table 2, the average evaluation accuracy of the method in this paper is $96.26 \%$, which is $5.36 \%$ and $14.29 \%$ higher than that of the BP neural network and gray target decision, respectively. Therefore, the PSO-LSSVM method has a very superior degree of accuracy and scientific performance.

Next, we performed 20 experiments on 10 datasets using the three methods, and the time used for the three methods (test time and training time) within the experiment was calculated while the average value was selected. The results of the comparison of the three methods are shown in Figures 5 and 6.

From the comparison results of Figures 3 and4, it can be seen that POS-LSSVM has obvious advantages over the other two methods in terms of time efficiency, the training time and testing time of this method are the lowest, the average training time is as low as $15 \mathrm{~ms}$, and the average testing time is as low as $5 \mathrm{~ms}$. Compared with the BP neural network, its training time saves $8 \mathrm{~ms}$ and its test time saves $5 \mathrm{~ms}$; compared with gray target decision, its training time saves $3 \mathrm{~ms}$ and its test time saves $4 \mathrm{~ms}$.

\section{Teaching Optimization Design}

The purpose of this paper is to come up with the most sensitive influencing factors through the constructed CETE evaluation model and then proposing some targeted ways to improve the effectiveness of English teaching. After further questionnaires and armor-piercing interviews, we conclude that $A_{11}, A_{12}, A_{14}$, and $A_{21}$ play a more important role for English teaching. The four indicators were moved up and down by $5 \%$ and $10 \%$, respectively, and the magnitude of the impact of each move on the evaluation results is shown in Figure 7. To facilitate the analysis of the degree of change in the evaluation results, the rate of change is expressed using the absolute value of the true value. Figure 7 illustrates that $A_{11}$ and $A_{14}$ have a higher degree of influence on the evaluation results compared to $A_{12}$ and $A_{21}$, which suggests that the optimization of CETE in this paper focuses on both course content and teacher skills [19].

In language learning, interests will be a great help to students. Therefore, we must find suitable methods to improve teachers' literacy and enrich teaching contents so as to stimulate the interest of student and improve the teaching effect [20]. Starting from the reality of STL, this paper mainly improves College English teaching through the integration of information technology and curriculum design.
4.1. Observation Dimension of Curriculum Design. Before the beginning of curriculum optimization, this paper observes the English curriculum of SIT colleges and universities, obtains research materials by means of field observation, and mainly observes the curriculum design of English teaching in SIT colleges and universities, the application of technical tools, and the integration of technology and curriculum content. The specific observation dimensions include teaching objectives, learning situation, teaching content, teaching media and curriculum materials, and teaching procedures.

In terms of teaching objectives, SIT teachers focus on cultivating college students' basic knowledge and skills of English audio visual according to the syllabus and curriculum requirements and pay attention to the cultivation of students' emotions, attitudes, and values in the teaching curriculum planning. However, it does not focus on cultivating students' English thinking and lacks the cultural elements in English function. In terms of learning, SIT teachers regard students as the learning center. In the whole process of English teaching, teachers pay enough attention to students' learning status and performance. However, as a freshman of SIT, the basic knowledge of English is not solid, and the knowledge structure is not systematic and perfect. Teachers' teaching rhythm is not synchronized with students' learning status and ability. As a result, teaching is hindered. In terms of teaching content, SIT teachers have the phenomenon of following the book, lack of effective design of teaching content, and no fine processing and expansion of knowledge and skills. The teaching content adheres to the textbook itself, resulting in the lack of interest in the course. In the application of teaching media and curriculum materials, SIT classrooms are equipped with advanced teaching technology tools, but teachers are not well applied to practical teaching, only as the presentation and display of curriculum materials, and curriculum design lacks theoretical guidance.

In addition, in the actual teaching process, teachers only use textbooks, markers, and whiteboards for teaching. Therefore, teaching technology cannot give full play to its maximum function, and it is in a state of separation from the whole teaching process. In terms of teaching procedures, SIT teaching procedures tend to be old, mainly including review, main curriculum, practice, summary, and home work There are some practical problems in this teaching procedure, which should be optimized to add links between teachers' activities and students' learning activities.

Thus, the application framework of teaching links, activity forms, and technical tools of the specific observation dimension of SIT English course is obtained, as shown in Figure 8. In the review session, the teacher summarizes and reviews the basic English knowledge and skills learned in the previous course, including cognition, understanding, and skills. In the main curriculum link, the teacher will present the prepared course content in writing and oral form according to the teaching requirements, mainly including theme introduction, vocabulary obstacle removal, key explanation, text explanation, and extracurricular knowledge expansion. In the practice link, teachers test the students' 
TABLE 2: Comparison of evaluation accuracy results.

\begin{tabular}{lccc}
\hline Dataset & PSO-LSSVM (\%) & BP neural network (\%) & Gray target decision (\%) \\
\hline 1 & 96.89 & 92.30 & 84.55 \\
2 & 95.72 & 88.76 & 83.43 \\
3 & 97.90 & 92.12 & 83.08 \\
4 & 94.34 & 93.62 & 80.89 \\
5 & 96.41 & 87.90 & 79.63 \\
6 & 98.02 & 89.54 & 82.60 \\
7 & 95.23 & 90.21 & 78.15 \\
8 & 96.29 & 92.88 & 83.42 \\
9 & 94.37 & 90.49 & 78.94 \\
10 & 97.46 & 91.25 & 85.03 \\
\hline
\end{tabular}

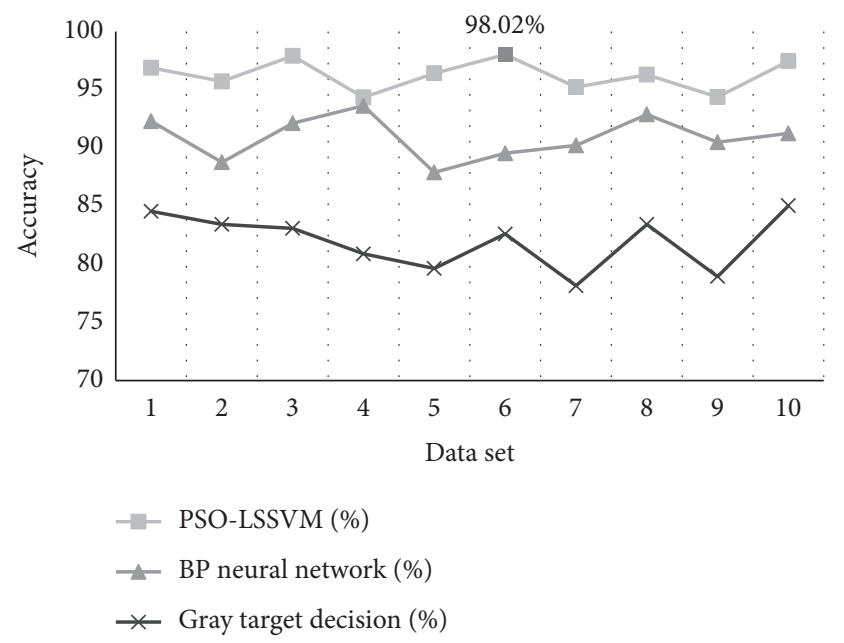

FIGURE 4: Comparison of evaluation accuracy results.

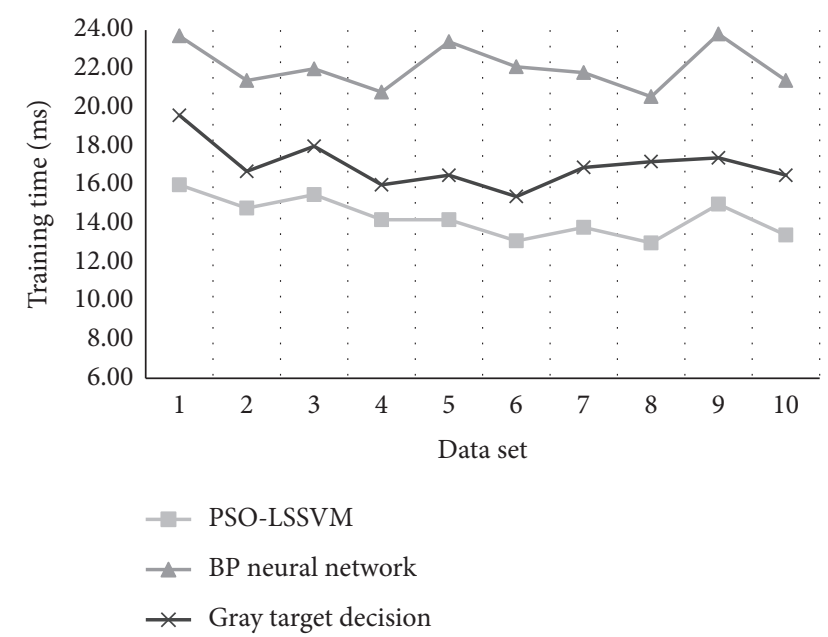

FIGURE 5: Comparison results of training time of different models.

learning of course knowledge in groups by random roll call and random inspection, and restate and explain the knowledge points with high error rate. In the summary section, teachers apply the consolidation method to review the course and repeat the key and difficult points of the course to understand the students' understanding of knowledge. In the home work link, the teacher assigns homework after class. The types of homework include

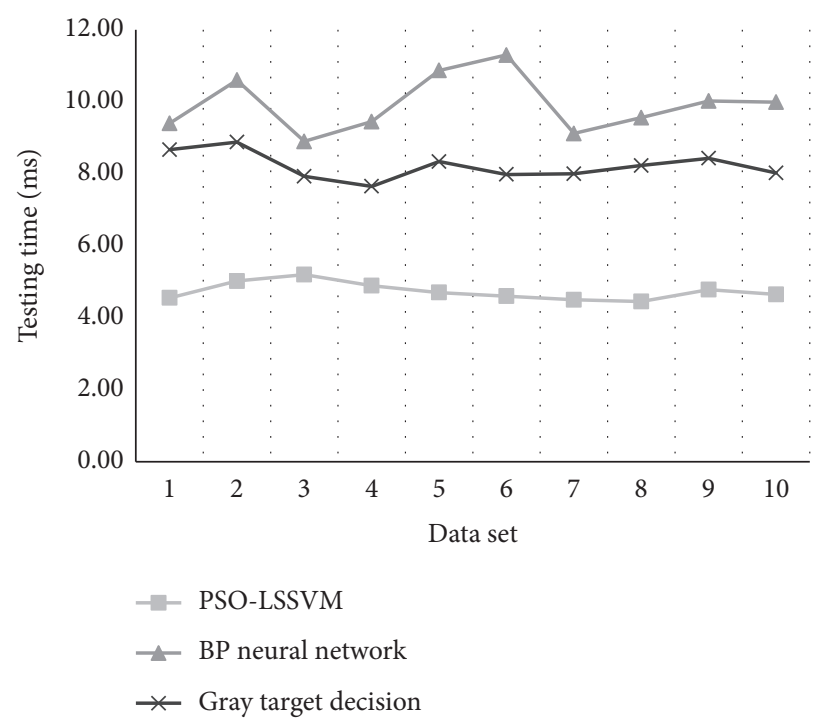

Figure 6: Comparison results of testing time of different models.

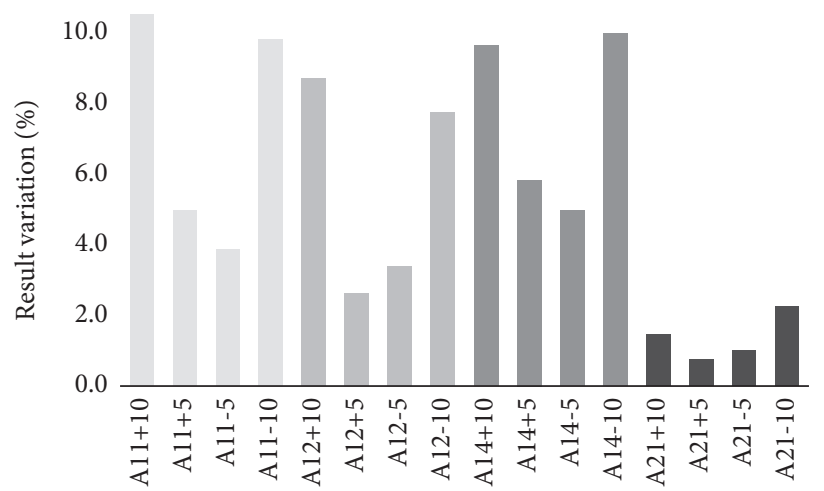

(\%)

Figure 7: Sensitivity analysis of important indicators.

dictation and recitation of key words, and flexible collocation and application of phrases and sentence patterns.

4.2. Optimization and Perfection of Teaching Procedure. This paper chooses an optimized program guided by the integration of information technology and curriculum, mainly to address the current situation of outdated teaching 


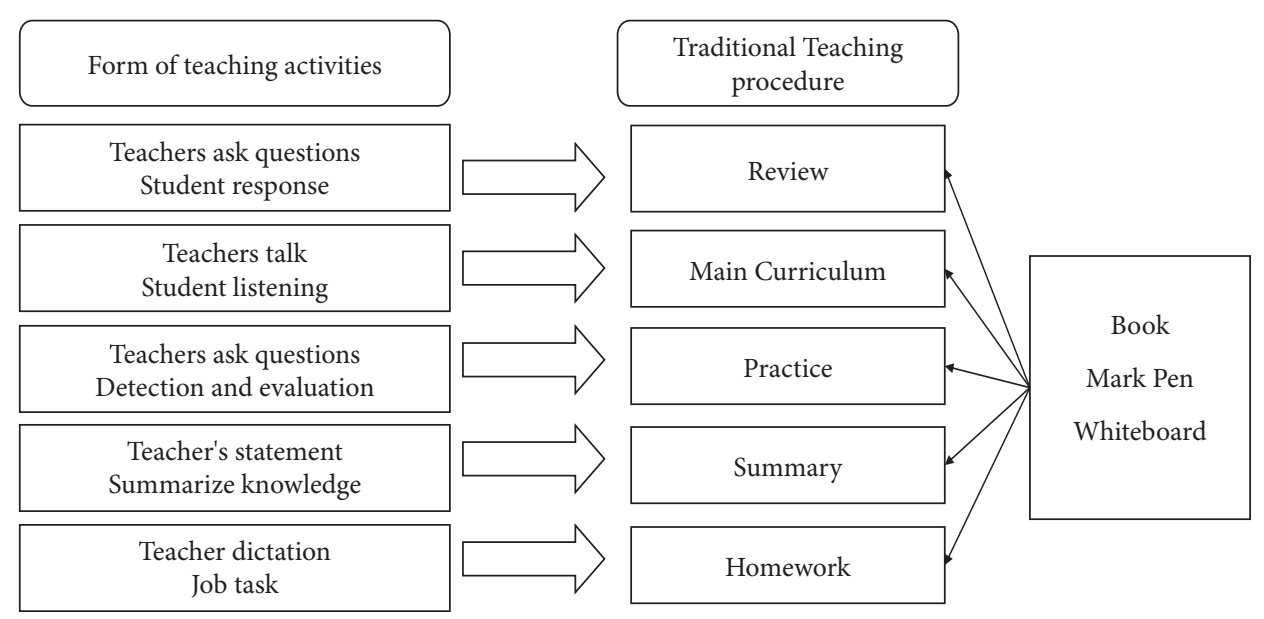

FIGURE 8: Teaching activity procedure of SIT universities before optimization.

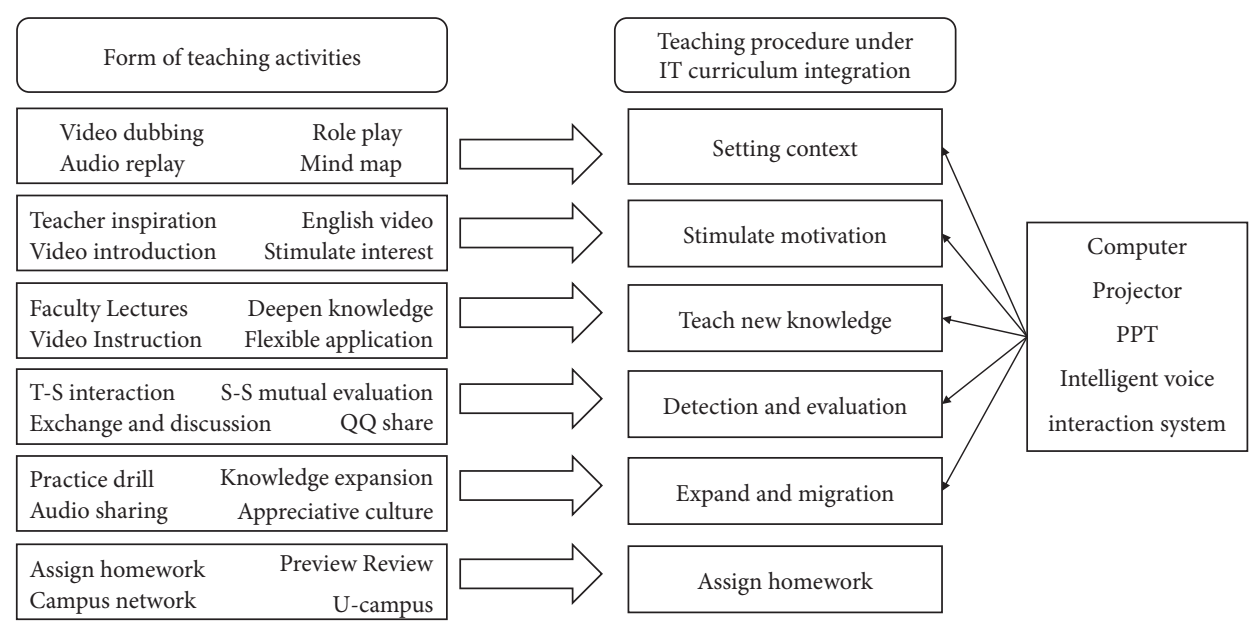

FIGURE 9: Teaching activity procedures under IT curriculum integration.

design, fragmentation, and disconnection between technological tools and actual teaching. The aim is to enhance the comprehensive ability of teachers, improve learning interest of students, and enhance the coherence of course content and teaching quality in future education and teaching. The main dimensions of teaching optimization design include the whole teaching process, using technology concepts and technology tools to integrate teaching dimensions. Every aspect of teaching is assisted and facilitated by the application of technology; the technology concept fully integrates the whole teaching process, the technology tools are no longer idle, and they are more efficiently integrated with teaching.

The intelligent voice system is integrated into all stages of the whole course teaching process to enrich the teaching content so that students can carry out role playing, audio and video appreciation, and other activities. This pedagogical optimization focuses on the teacher-led-student-subject role in the teacher-student teaching role position dimension. Students learn under the inspiration, guidance, and teaching of the teacher. Then, under the guidance of this theory of teaching-learning relationship, the optimization of teaching and learning under the guidance of IT and curriculum integration is achieved through the use of technological tools, pedagogical aids, and the integration of subject content concepts into the whole teaching process. In general, the study provides a careful design and detailed analysis of the procedures of teaching activities so that the optimized teaching design can be more effective. The specific contents of teaching optimization and the integrated application of information technology are shown in Figure 9.

\section{Conclusion}

The quantitative nonlinear functions between the CETE and each evaluation index are complicated. The complex mathematical relationships lead to subjectivity in the evaluation scores, which affects the objectivity and fairness of the evaluation. In order to accurately evaluate the effectiveness of College English teaching and improve the overall level of College English teaching, this paper studies the evaluation model of College English teaching effectiveness based on PSO-LSSVM. The average accuracy of the evaluation model in ten datasets is $96.26 \%$, which has high advantages and is nearly $6 \%$ higher than that of the BP neural network. The average training time is $15 \mathrm{~ms}$, and the average test time is 
$5 \mathrm{~ms}$. The experimental results show that this method has the highest evaluation accuracy, short evaluation time, and the best evaluation results. Based on the method of this paper, it can improve the optimization plan for English teaching effect, which is of great practical significance for the improvement of College English teaching level.

During the implementation of this study, the concept of information technology and curriculum integration is applied to guide teaching and learning and to carry out teaching practices. This a priori concept optimizes and improves the whole teaching system, and each component of the teaching system incorporates the concept of technology integration. At the same time, it focuses on the integration of technology, pedagogy, and subject content. As a result, SIT is optimized, and students' English listening and speaking skills are improved.

Due to the limited time and energy, there are still some deficiencies to be further improved. Firstly, in order to improve the generalization of the research, we need to try to apply better methods to the in-depth study of College English teaching evaluation index system in future research so as to make it complete and more scientific. Secondly, more experimental datatests are carried out for the method in this paper, and the method is continuously optimized in the test. Finally, it is hoped that the in-depth study will apply better methods to College English teaching and make the evaluation accuracy and efficiency better.

\section{Data Availability}

The labeled dataset used to support the findings of this study is available from the corresponding author upon request.

\section{Conflicts of Interest}

The author declares no conflicts of interest.

\section{References}

[1] G. Eleni, P. Eugenia, and C. Dora, "Identifying factors of job motivation and satisfaction of foreign language teachers: research project," Procedia-Social and Behavioral Sciences, vol. 46, no. 5, pp. 14-29, 2012.

[2] Y. Li, M. Yu, and Y. Hu, "Modeling and analysis of classroom teaching evaluation platform based on colored petri nets," in Proceedings of the IEEE International Conference on Electronics Information and Emergency Communication, Beijing, China, July, 2019.

[3] D. Nalla, "A framework for teaching evaluation," in Proceedings of the 2018 IEEE Frontiers in Education Conference (FIE), San Jose, CA, USA, October, 2018.

[4] C. K. Leong, Y. H. Lee, and W. K. Mak, "Mining sentiments in SMS texts for teaching evaluation," Expert Systems with Applications, vol. 39, pp. 2584-2589, 2012.

[5] T. Ahmad, "Teaching evaluation and student response rate," PSU Research Review, vol. 2, no. 3, pp. 206-211, 2018.

[6] S. Cadez, V. Dimovski, and M. Zaman Groff, "Research, teaching and performance evaluation in academia: the salience of quality," Studies in Higher Education, vol. 42, no. 8, pp. 1455-1473, 2017.
[7] H. H. Bi, "A robust interpretation of teaching evaluation ratings," Assessment \& Evaluation in Higher Education, vol. 43, no. 1, pp. 79-93, 2018.

[8] T. Mullen and N. Collier, "Sentiment analysis using support vector machines with di-verse information sources," in Proceedings of the 2004 Conference on Empirical Methods in Natural Language Processing, pp. 23-35, Barcelona, Spain, July, 2004.

[9] R. Moraes, J. F. Valiati, and W. P. Gavião Neto, "Documentlevel sentiment classification: an empirical comparison between SVM and ANN," Expert Systems with Applications, vol. 40, no. 2, pp. 621-633, 2013.

[10] J. Kennedy and R. C. Eberhart, "A discrete binary version of the particle swarm algorithm," in Proceedings of the IEEE International Conference on Systems, Man and Cybernetics, pp. 4104-4108, IEE Service Center, Orlando, FL, USA, October, 1997.

[11] Z. M. Sun, M. Anbarasan, and D. P. Kumar, "Design of online intelligent English teaching platform based on artificial intelligence technique," Computational Intelligence, vol. 37, no. 9, pp. 1-15, 2020.

[12] R. A. Krohling and T. T. M. de Souza, "Combining prospect theory and fuzzy numbers to multi-criteria decision making," Expert Systems with Applications, vol. 39, no. 13, Article ID 11487, 2012.

[13] W. Lan, "Student model construction of intelligent teaching system based on Bayesian network," Personal And Ubiquitous Computing.vol. 24, no. 3, pp. 419-428, 2020.

[14] M. H. Myers, “Automatic detection of a student's affective states for intelligent teaching systems," Brain Sciences, vol. 11, 2021.

[15] R. A. Ramadan and A. V. Vasilakos, "Brain computer interface: control signals review," Neurocomputing, vol. 223, no. 5, pp. 26-44, 2017.

[16] S. Mehrkanoon, T. Falck, and J. A. K. Suykens, "Approximate solutions to ordinary differential equations using least squares support vector machines," IEEE Transactions on Neural Networks and Learning Systems, vol. 23, no. 9, pp. 1356-1367, 2012.

[17] Y. Luo, X. F. Zhao, and Y. Y. Qiu, "Evaluation model of art internal auxiliary teaching quality based on artificial intelligence under the influence of COVID-19," Journal of Intelligent and Fuzzy Systems, vol. 39, no. 6, pp. 8713-8721, 2020.

[18] Y. Feng, "An evaluation method of PE classroom teaching quality in colleges and universities based on grey system theory," Journal of Intelligent and Fuzzy Systems, vol. 38, no. 6, pp. 1-12, 2020.

[19] A. Miner, J. Mallow, L. Theeke, and E. Barnes, "Using gagne's 9 events of instruction to enhance student performance and course evaluations in undergraduate nursing course," Nurse Educator, vol. 40, no. 3, pp. 152-154, 2015.

[20] F. X. Ma, Z. C. Zhu, and M. Zhou, "Fuzzy comprehensive evaluation of classroom teaching quality of college teachers," Jinternational Journal of Electrical Engineering \& Education, 2020. 